\title{
A Practical Method for Tradespace Exploration of Systems of Systems
}

\author{
Debarati Chattopadhyay $^{*} \quad$ Adam M. Ross ${ }^{\dagger}$ \\ Donna H. Rhodes $\ddagger$ \\ Massachusetts Institute of Technology, Cambridge MA 02139
}

\begin{abstract}
Systems of Systems (SoS) are a current focus of many organizations interested in integrating assets and utilizing new technology to create multi-component systems that deliver value over time. The dynamic composition of SoS along with the managerial independence of their component systems necessitates systems engineering considerations and methods beyond those of traditional systems engineering, particularly during SoS concept design. Qualitative and heuristic-based guidance for SoS design is available in the literature, but there is a need for methods that will allow decision makers to quantitatively compare diverse multi-concept SoS designs in order to select value robust designs during concept exploration. In this paper, a quantitative method for SoS conceptual design, known as System of Systems Tradespace Exploration Method (SoSTEM), is presented. This method is based on the existing Dynamic Multi-Attribute Tradespace Exploration (MATE). MATE is a formal methodology for tradespace exploration during system design that allows the decision maker to make trades between both stakeholder preferences and systems early in the design process and includes the consideration of dynamic issues such as unarticulated stakeholder preferences and changing system context. SoSTEM enables the generation of SoS tradespaces where multi-concept architectures can be compared on the same performance and cost basis. This method allows the SoS designer to distinguish between component systems having high likelihood of participation in the SoS and those with lower likelihood of participation, based on the level of 'Effective Managerial Authority' that the SoS designer has over the component. In this paper, SoSTEM is demonstrated through application to two case studies, an Operationally Responsive System for Disaster Surveillance and a Satellite Radar System.
\end{abstract}

\section{Introduction}

Systems of Systems (SoS) are dynamic, higher-order systems that are composed of other independently managed component systems. SoS are of increasing importance as organizations today use highly networked but independently managed systems to generate unique capabilities beyond that available by independent operation of those systems. Due to the additional complexity of component systems that are independently managed, SoS engineering requires different considerations when compared to traditional systems engineering.

As the majority of engineering resources are committed in the concept exploration phase of systems engineering, one of the primary challenges in engineering system design is making decisions in the concept exploration phase that will result in designs that are valuable throughout the operational lifetime of the system. The problem is even more difficult when designing SoS, which often change in composition several times during the SoS lifetime, incorporating a different combination of both legacy and newly designed component systems at different times during the SoS operational life. Tradespace exploration methods are employed in system design to analyze design spaces and aid decision-makers in choosing 'good' design alternatives from among a potentially large set. As the emphasis on designing SoS is increasing, there is a

* Research Assistant, Department of Aeronautics and Astronautics, 77 Massachusetts Avenue, Cambridge MA 02139.

${ }^{\dagger}$ Research Scientist, Engineering Systems Division, 77 Massachusetts Avenue, Cambridge MA 02139. AIAA Member

¥Senior Lecturer, Engineering Systems Division, 77 Massachusetts Avenue, Cambridge MA 02139. AIAA Member 
need for a tradespace exploration methodology that will allow designers to make informed design decisions early in the SoS conceptual design phase, as well as make informed operations and management decisions during operation of SoS. The System of Systems Tradespace Exploration Method (SoSTEM) is proposed as a method to generate and analyze tradespaces to support decision making in the SoS conceptual design phase. SoSTEM is then applied to two case studies in order to demonstrate the identification of value robust SoS designs and illustrate the design insights that can be obtained using this method.

\section{Literature Review}

Literature related to descriptions of Systems of Systems, the classification of SoS, and the need for new systems engineering methods for SoS design has been a growing field. There are several definitions of SoS that are currently available in the literature, though there is no commonly agreed upon definition. INCOSE ${ }^{1}$ defines Systems of Systems as

System of Systems applies to a system-of-interest whose system elements are themselves systems; typically these entail large scale inter-disciplinary problems with multiple, heterogeneous, distributed systems.

A variety of descriptions of SoS have also been put forth in the literature, with characteristics such as Operational and Managerial Independence from Maier ${ }^{2}$; Evolutionary Development and Geographical Distribution from Sage and Cuppan ${ }^{3}$; Autonomy, Belonging, Connectivity, Diversity and Emergence from Boardman $^{4}$. While these characterizations are either very detailed or broad enough to include a wide range of systems within their purview, there are a few traits common to most of the SoS descriptions, which can be summarized in the following statement - that SoS are systems, composed of a set of heterogeneous systems that maintain some level of independence while within the SoS and provide some emergent value within the SoS beyond their individual contributions.

From the characteristics differentiating SoS and traditional systems in the literature, it is evident that SoS have additional complexity compared to traditional systems. Due to this added complexity, there is a possible need for improved, or in some cases, new systems engineering methods to design SoS. Eisner ${ }^{5}$ and Keating ${ }^{6}$ describe the need for SoS Engineering methods beyond traditional systems engineering methods. Several heuristics (such as those proposed by $\mathrm{Maier}^{2}$ and $\mathrm{Sage}^{3}$ ) and qualitative frameworks for SoS engineering (as discussed by Carlock ${ }^{7}$ and DeLaurentis ${ }^{8}$ ) have been proposed to address SoS design. While qualitative frameworks provide much-needed guidelines for development of more rigorous methods for SoS design, there is still a need for more quantitative methods for SoS design. Recently there has been some research development in this area, using modeling and simulation to generate the performance of many SoS designs for quantitative comparison.

\section{Motivation}

A quantitative method of SoS conceptual design will enable the comparison of many more architecture options than is possible through qualitative methods alone, facilitating a more complete exploration of a SoS design space. Such a quantitative method needs to address the SoS-specific characteristics that distinguish SoS engineering from traditional systems engineering, and enable the decision maker to compare a large number of SoS designs consisting of heterogeneous components on an equal basis in order to aid the decision maker in selecting value robust SoS designs.

Tradespace exploration is a method that has been used in the conceptual design of traditional single systems in order to quantitatively compare a large number of design options on the same performance and cost basis. Extending an existing system tradespace exploration method to the SoS domain will lead to a quantitative SoS conceptual design method. In this paper, a SoS tradespace exploration method based on the existing Dynamic Multi-Attribute Tradespace Exploration (MATE) method ${ }^{9}$ is presented to address the need for a quantitative SoS conceptual design method to aid SoS decision makers.

\section{Identification of SoS-specific Design Considerations}

To develop the SoS Tradespace Exploration Method, several SoS-specific design characteristics were identified that distinguish SoS conceptual design from that of traditional systems. 


\section{A. Stakeholder Analysis Differences for SoS}

SoS are composed of component systems that maintain some level of independence while participating in the SoS. A component system has independent management that makes decisions about the system operation, both when the system is participating in and also when it is outside of the SoS. By virtue of being systems themselves, component systems have their own sets of stakeholders. Some of these local stakeholders may also be part of the global SoS stakeholder set. Figure 1 illustrates this concept of local and global stakeholders.

Due to stakeholder sets at both the local component system level and global SoS level, a SoS designer is confronted with a multi-level stakeholder value proposition with multiple stakeholders at each level during stakeholder analysis. Multistakeholder negotiations may require aggregating and trading the preferences of decision makers, depending on the relations between the local and global stakeholders. The designer must incorporate local and global distribution of costs and benefits into a multi-level value proposition for the SoS. In order to design a SoS that will be able to deliver the expected value to the SoS stakeholders, the SoS designer must take into account the independent decision making capability of each component system resulting in the risk of non-participation of a component when it is needed as well as strategies to maintain the desired SoS configuration by influencing the behavior of the SoS component systems.

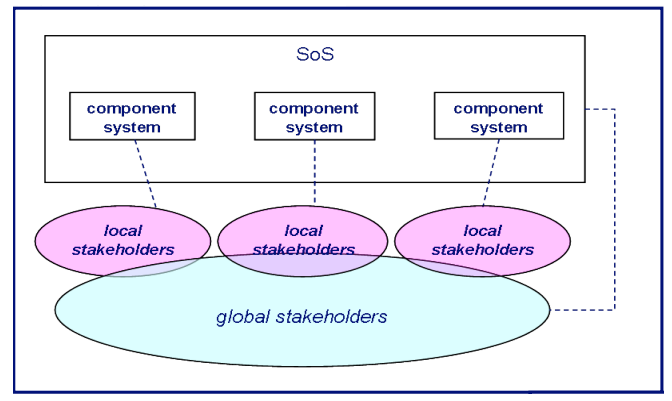

Figure 1. SoS Local and Global Stakeholders, resulting in a multi-level stakeholder value proposition. Component systems have local stakeholders; the SoS has global stakeholders. Some local stakeholders may also be part of the global stakeholder set.

\section{B. Dynamics of SoS Composition}

SoS face changing conditions over time, such as component systems joining and leaving the SoS, changes in component systems available for inclusion in the SoS, various stakeholder preference changes, and external context changes that may affect the value delivery of the SoS.

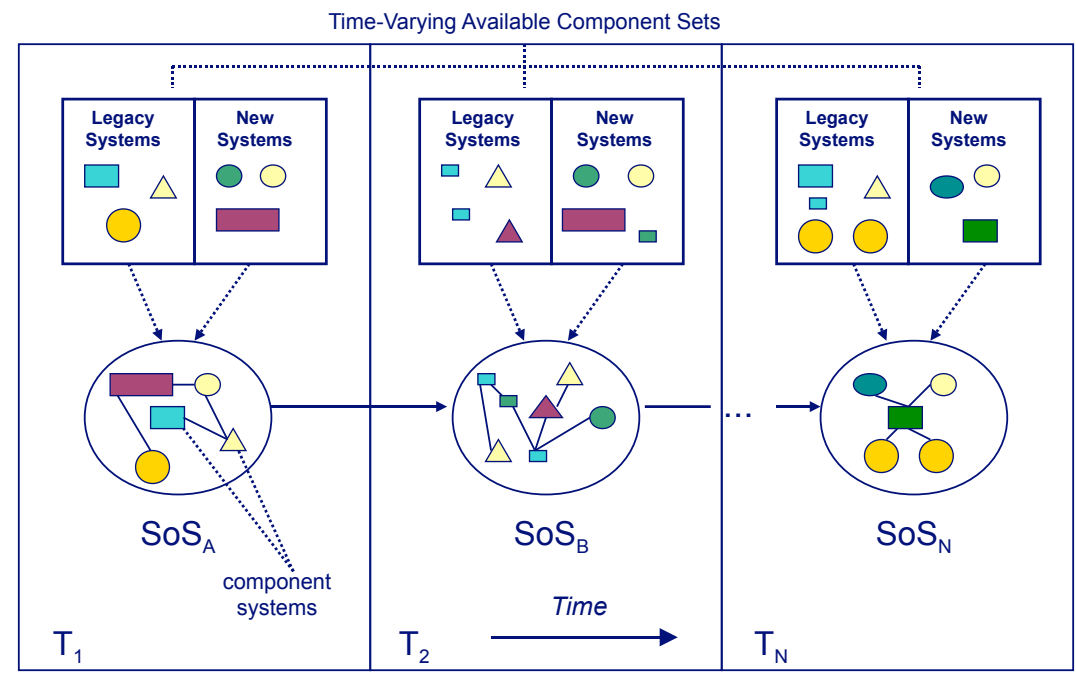

Figure 2. Legacy and New Component Systems in Time-Varying SoS Composition

Figure 2 is a notional diagram representing the potential changing SoS composition, as well as the incorporation of legacy and new component systems within the SoS. It is important to identify value robust SoS designs (which are designs that maintain a certain level of value delivery under changing conditions) during the design process in order to aid the selection of designs that will remain useful over the SoS operational lifetime. Epoch-Era Analysis is an approach applied in Dynamic MATE to analyze systems that operate in a time-varying context ${ }^{9}$ and may help identify value robust SoS designs in the conceptual design 
phase. In Epoch-Era Analysis, the SoS lifetime can be divided into a series of epochs, which are defined as time periods when significant system design characteristics, expectations, and context variables are fixed. Multiple consecutive epochs can be strung together to create an era, which represents a longer run view of the system evolution. Significant changes in the SoS or the SoS context - such as a component system joining or leaving the SoS - can be represented by defining a new epoch. Since SoS are often created to meet an urgent need, evolutionary development may be needed to build up capability over time through different configurations of component systems within the SoS. While a single epoch can give a SoS designer an idea of SoS designs that are valuable in a particular fixed context, arranging multiple epochs into longer periods called eras can provide a long-term view of the SoS evolution and help the SoS designer identify long-term strategies for sustaining the SoS.

\section{Legacy and New Component Systems}

SoS are often composed of both legacy and new systems, as well as existing and newly-designed interfaces between component systems. The SoS designer may not have the ability to affect enhancements and upgrades to legacy systems or interfaces. The 'system shell' concept ${ }^{10}$ may be a useful construct when the component system design cannot be altered. By designing a wrapper or shell around the legacy component, it can easily be integrated into the SoS and interfaced with other components without adversely affecting the legacy operation. This concept may also make it easier to switch components in and out of a SoS with minimum impact on the SoS operation. On the other hand, the SoS designer may have the power to define the design of a system that is being newly designed. Thus the SoS designer must take into account the differences in design control over legacy and new component systems during decision making in the conceptual design process.

\section{Managerial Control, Influence and Participation Risk}

As discussed in the previous section, the level of design control that the SoS designer effectively has over each component will affect the ultimate design of the SoS. To address this issue of SoS designer control over components, a framework for decision making that drives the participation of desired component systems in a SoS was developed. A SoS is comprised of component systems that retain some level of independent management and operation. The relationship between the SoS designer, responsible for SoS-level decisions, and the component system management, responsible for component system decisions, can be represented with two quantities - Managerial Control and Influence. Managerial Control is the amount of hierarchical control that the SoS designer has over the component system. Managerial Control has been previously used to classify SoS in the literature, for example by Maier. ${ }^{2}$ In cases where participation of component systems is voluntary, the SoS designer may employ various methods, collectively referred to as Influence, in order to persuade component systems to behave in a way that is beneficial to the SoS. The component system management may make decisions about the component system participation in the SoS based on their local perception of benefits and costs of participation. The change in 'Perceived Net Benefit' (PNB), which is the difference between local benefit and local cost as perceived by the component system management, is a metric that can be used by the component system management to determine whether to join the SoS. The SoS designer may be able to change the PNB for a component system by offering incentives or additional non-monetary value to influence the component system behavior. ${ }^{11}$

The 'Effective Managerial Authority' (EMA) that a SoS designer has over the component systems is composed of the Managerial Control described above, and the Influence exerted by the SoS designer to change the PNB of the component system, as shown in Figure 3. A greater EMA over a component system leads to greater likelihood of participation of the component system in the SoS. The risk of non-participation of a component system in the $\mathrm{SoS}$ is defined as the 'Participation Risk' (PR) of the component system, from the perspective of the SoS designer. Participation Risk is defined in Equation 1. In Figure 3, Participation Risk is shown by the 'Gap' indicated.

$$
\begin{aligned}
\text { Participation Risk } & =1-(\text { Effective Managerial Authority }) \\
& =1-(\text { Control }(M C)+\text { Influence }(\text { In })) \text { where } 0 \leq(M C+I n) \leq 1
\end{aligned}
$$




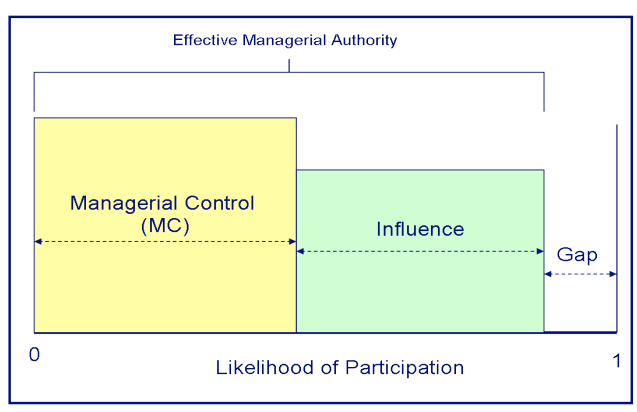

Figure 3. Effective Managerial Authority, Composed of Managerial Control and Influence. The 'Gap' Represents Participation Risk.
Participation Risk represents the uncertainty involved in attaining a particular desired SoS design configuration. PR allows the SoS designer to differentiate between easy to achieve SoS designs and relatively difficult to achieve SoS designs in the tradespace. In the SoS tradespace, PR can be used as a way to filter out difficult to achieve SoS designs. This information allows an SoS decision maker to compare designs on the basis of performance and cost as well as the risk of not achieving those performance and cost values.

The PR of the component systems can be estimated by the SoS designer, based on approximations for the designer's Managerial Control over the component and the estimated Influence, either monetary or otherwise, that the designer expects to have over the component. In this method, the Effective Managerial Authority (and the PR derived from it) is a variable for that particular component. EMA can be varied over a range generating many SoS designs which can them be plotted on the tradespace.

The PR for a particular SoS design can be derived from the PR estimates of the component systems in the SoS. For example, if the PR for component system A is 0.8 and PR for component system B is 0.2 , the corresponding 'Likelihood of Participation' (LP) for each is $(1-P R)$, or 0.2 and 0.8 for A and B, respectively. Assuming that the participation of components is independent of each other, the combined Likelihood of Participation for the SoS is the product of the individual components' LP, i.e. $0.2^{*} 0.8=0.16$. Therefore, the combined PR for the SoS design AB would be $(1-L P)=0.84$. This PR for each SoS design allows the designer to compare within the tradespace the relative likelihood of achieving diverse SoS configurations. The designer can identify high performance, low cost designs in traditional tradespace exploration, but the newly added PR component enables the designer to also differentiate between configurations that are easy to achieve versus those that are relatively difficult to arrange. This enables the SoS designer to compare high performance-high risk designs with relatively lower performance-low risk designs that may be a more attractive solution.

\section{Combining SoS Attributes to Quantify SoS Value Delivery}

An integral part of the development of SoSTEM is the ability to estimate, in the concept exploration phase, the value generated by potential SoS designs. The SoS system value delivery is a function of the value delivery of the component systems, but is also greater than just the aggregation of the component system performance. Within the MATE framework, system attributes are decision maker perceived metrics used to measure the system value delivery to stakeholders. In SoSTEM, the component system attributes, as well as information about the concept of operations of components within the SoS, is used to model the SoS performance attributes. The classification of the component system attributes and the level of complexity of combination of the attributes is used to estimate the SoS integration cost. Combining attributes to model SoS value and estimate SoS cost impacts in order to generate SoS utility-cost tradespaces is a key aspect of SoSTEM.

According to Ross, ${ }^{12}$ system attributes can be classified on the basis of whether they are articulated by the decision maker, as well as the cost to display the attribute in the system. Attributes are classified into five categories by Ross, ${ }^{12}$ ranging from Class 0 to Class 4 . Class 0 attributes are those that are traditionally considered in system design and are exhibited by the system by intentional design, whereas Class 1 attributes represent the existing latent value in the system, or the additional attributes beyond the Class 0 attribute set that can be utilized by the system designer at no additional cost. Class 2 attributes can be generated through combination of traditional component system attributes and system latent value, while Class 3 attributes are attainable through some change in the system at acceptable cost. Class 4 attributes are only available through drastic changes of the original system at prohibitive cost, or not achievable at all given the physical constraints of the original design and are thus not available to the SoS designer. The superset of attributes can be generated by combining the Class 0,1,2 and 3 attributes of all of the component systems available for the SoS provides the set of attributes that can potentially be displayed by the SoS design. Each SoS attribute is generated through a combination of one or more of the attributes in this superset. 
As Class 0 and 1 attributes are more readily available to the SoS designer than Class 3 attributes, which require modification of or addition to the systems at some cost, SoS attributes that utilize Class 0 and Class 1 attributes of the component systems are more easily achievable compared to those that require Class 3 component system attributes.

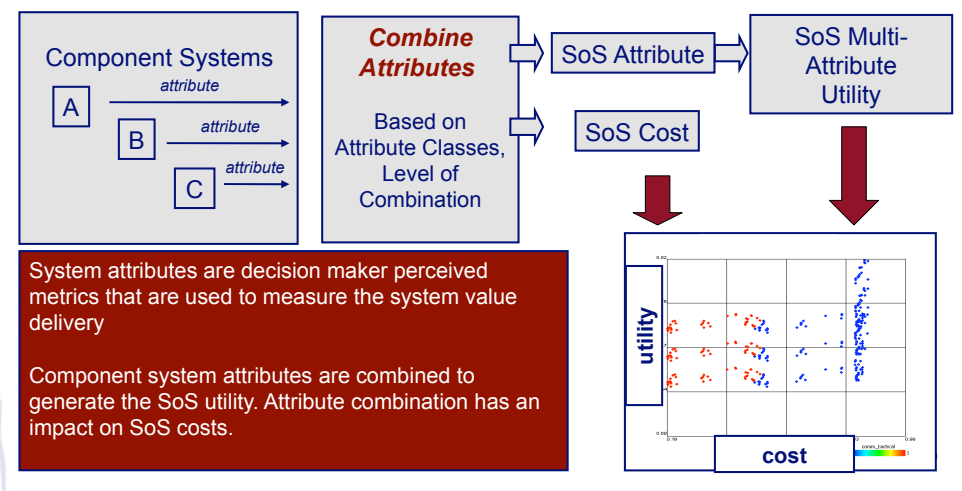

Figure 4. Combining Component Attributes to Obtain SoS-level Attributes in SoSTEM

Component attributes can be combined to generate a SoS-level attribute by selecting a level of combination complexity, effectively approximating the complexity of the interface required between components to generate the SoS attribute in the absence of more detailed information early in the design phase. The way in which the SoS components interact during SoS operation is an integral consideration in determining the interfaces between the components and also in determining how component attributes can be combined to achieve the SoS attributes. In early concept exploration, there are few constraints on the design space, so the SoS designer needs to explore the possible methods of attribute combination to determine which type is suitable for the particular SoS design. As this space of possibilities is large, as a first-order estimate, three types of attribute combination methods can be defined: low level, medium level and high level complexity. It is assumed that the higher the complexity of attribute combination, the higher the cost for creating an interface capable of this kind of combination. Low level methods may involve taking the best performance in a particular SoS attribute from the set of components in the SoS. If the SoS attributes are such that the mission is differentiated between the components (in other words, each component system provides a unique subset of attributes), then this may be the level of attribute combination that is required. Medium level attribute combination is required when there are more complex SoS concepts of operation. For example, when there is a handoff between different assets in the SoS, such that multiple components are involved in delivering a single attribute performance, the resulting SoS attribute performance is a combination of the two component attribute performances. Methods used for SoS attribute combination at the medium level may involve time-weighted averaging, such as the concept of time-weighted average utility. ${ }^{13}$ The highest level of attribute combination is required when multiple SoS components deliver performance relating to the same SoS attribute simultaneously. In this case, fusion of the attributes at a more detailed level than just averaging is required. A possible set of methods for combination of attributes at this level is data fusion. Data fusion is a well-developed field with methods available for combining data-related attributes, such as image resolution. Data fusion is defined as the combining of data from multiple sources along with database information to draw inferences beyond that obtainable through a single data source alone. ${ }^{14}$ The SoS designer can select a particular data fusion method for attribute combination in order to obtain the SoS attribute from multiple component sensors.

Once the method to combine attributes, dependent on the level of combination complexity chosen, is selected, the SoS attribute value is obtained as a function of the component system attributes.

$$
\text { SoS attribute value }=f n(\text { componentA attribute, componentB attribute, componentC attribute...) }
$$

The described framework of combining attributes is utilized within SoSTEM to quantitatively model the SoS performance using any set of component systems, and enables the consideration of diverse multi-concept design configurations on the same tradespace. Figure 4 shows the steps of component system attribute combination leading to quantification of SoS utility in order to generate SoS tradespaces in SoSTEM. 


\section{System of Systems Tradespace Exploration Method (SoSTEM)}

The SoS Tradespace Exploration Method (SoSTEM), pictorially represented in Figure 5 , was developed as a method to aid decision makers in SoS tradespace exploration in the early conceptual design phase. The concepts of Participation Risk of components, combination of component system attributes to generate SoS attributes and studying the time-varying SoS value delivery through multi-epoch analysis are integrated into a quantitative method for SoS conceptual design that enables the modeling and comparison of a large number of design alternatives.

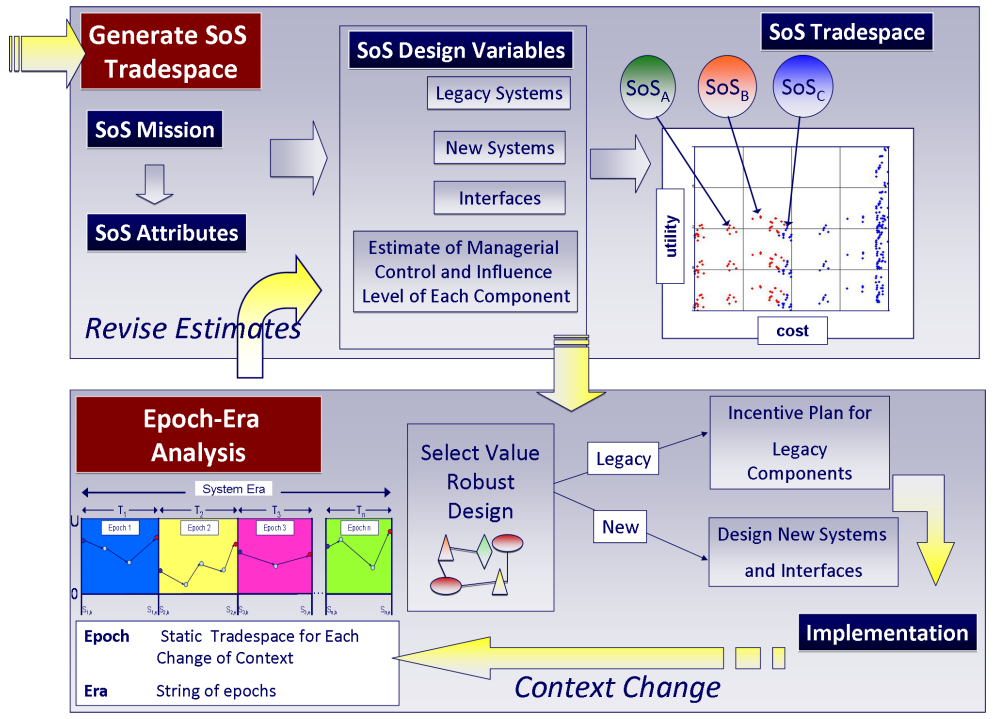

Figure 5. SoS Tradespace Exploration Method (SoSTEM)

The ten steps of the method are described in Table1.

\section{Case Applications}

Aspects of SoSTEM were developed through application to a case study that involved the conceptual design of a Operationally Responsive Disaster Surveillance System. This was followed by the application of SoSTEM to the conceptual design of a Satellite Radar System.

\section{A. Operationally Responsive Disaster Surveillance System}

The Operationally Responsive System for Disaster Surveillance is an example case to demonstrate the usefulness of the SoS tradespace exploration method in dealing with multi-stakeholder and multi-concept design problems, and to show that even with limited resources and functional modeling, this method can provide practical insights to the SoS decision maker. The case study research was conducted by a team of researchers from MIT and the Charles Stark Draper Laboratory. Given the numerous severe natural disasters in the recent past, such as Hurricane Katrina in New Orleans in 2005, the California wildfires of 2007 and 2008, and the Sichuan earthquake in China in 2008, there is a clear need for effective and timely observations of disaster locations in order to aid first responders. For disaster surveillance information to be of use to first-response and disaster relief efforts, it must be provided as soon as possible after unforeseen events in unknown locations. In other words, an operationally responsive system with short response time is necessary to generate disaster observing data that is useful for time-critical disaster relief.

To address the question of multiple SoS stakeholders, two SoS stakeholders - a firefighter and the Operationally Responsive System owner - were simultaneously considered in the stakeholder analysis. Heterogeneous SoS consisting of multiple single system concepts, both legacy and new, were compared on the same performance and cost basis in a tradespace, alongside single system concepts. Finally, the analysis of the effects of dynamic context changes including changes in stakeholder preference on the system through Epoch-Era Analysis was used to identify potentially value robust designs. While the full SoS tradespace ex- 
AIAA Space 2009

Pasadena, CA, September 14-17, 2009

Step
Determining the SoS Mission
Generating a List of Component Systems
Identifying Stakeholders and Decision Makers
for SoS and Component Systems

Classifying Component Systems According to Managerial Control and Participation Risk

Defining SoS Attributes and Utility Information

Defining SoS Context Changes

Modeling SoS Performance and Cost

Tradespace Analysis

Epoch-Era Analysis

Selecting Value Robust SoS Designs
Description

The SoS mission is the problem that the SoS must solve or capability gap that the SoS must fulfill, generated through interaction with the SoS stakeholders.

A list of legacy systems that may fully or partially fulfill the mission need is generated, and new system concepts can be proposed to fill any capability gaps. The output of this step of the method is a list of potential component systems for the SoS.

Local component system stakeholders and SoS level stakeholders are identified. In SoSTEM, it is assumed that the SoS and each legacy component system have a single decision maker each - this may require an aggregation of stakeholder preferences by defining a benevolent dictator. The benevolent dictator is a primary decision maker who takes actions to allocate resources in such a way as to satisfy the stakeholder network he/she represents.

The identification of the primary decision-maker for the SoS and each of the potential component systems enables the SoS designer to estimate the amount of managerial control that the SoS decision-maker has over each of the component systems. This is necessarily an iterative step, as the estimation of managerial control may not be accurate at this stage, due to limited available information.

Attributes (performance metrics used to measure system value delivery to the stakeholders) and utility (used to represent aggregate stakeholder satisfaction based on system performance on the tradespace) information is obtained through interviews of the SoS stakeholder.

A number of possible future context changes, such as SoS stakeholder preference changes, changes in control and participation risk, and changes in availability of component systems for inclusion in the SoS, are identified in order to study the changes in SoS value delivery over time in Epoch-Era Analysis. Includes modeling legacy systems as well as clean-sheet systems, as well as modeling the SoS in terms of utility and cost in order to generate tradespaces where each design is represented in terms of performance and cost.

Tradespaces including both single system designs and multiconcept SoS can be analyzed and the Pareto Set of designs can provide a means to select SoS designs that are suitable for further study.

The models created for the SoS performance calculation can be repeatedly run for each future epoch described by the context changes defined earlier in the method. Thus many tradespaces are obtained, and tradespace statistics can be run over a string of epochs which compose an era. An era represents a potential future scenario for the SoS.

Tradespace statistics such as Pareto Trace Number can be used to select designs that are value robust over multiple epochs, or eras.

Table 1. Description of the Steps of the System of Systems Tradespace Exploration Method

ploration method was not applied to this case study, the demonstration of significant aspects of the method through the case study demonstrates the practical usefulness of aspects of the method as a prescriptive tool for decision analysis during early conceptual design.

Three different single system concepts were considered in the first application of the method to the case - satellites, aircraft and sensor swarms. Using SoSTEM, the component systems performance and cost could be modeled individually, and then through combining attributes, SoS consisting of multiple single systems (such as an aircraft and a satellite together) could also be modeled. The single systems and multi-concept SoS were then represented on the same tradespace. A second application of SoSTEM was used with more detailed aircraft and satellite models, and multi-epoch analysis was conducted with a number of potential context changes. For example, a change in the location of the target area of interest may occur during the

$$
8 \text { of } 14
$$


system lifetime. In the case of this disaster surveillance system, it is expected that the system will need to observe a large number of different types of disasters on different locations on the globe during its operational lifetime. To demonstrate the application of SoSTEM to this type of context change, three different scenarios were considered in this analysis a) a hurricane disaster area, modeled on the Hurricane Katrina disaster area, b) a forest fire disaster, based on the Witch Creek Fire in California, and c) a cyclone disaster area, associated with the Myanmar cyclone in 2007.

Figure 6 shows an example of three epochs in which the target surveillance area is the context change under consideration. Tradespace statistics such as Pareto Trace Number ${ }^{15}$ are then used to identify passively value robust designs among the single system and SoS design concepts, which are then used as the set of designs to consider in further detail.

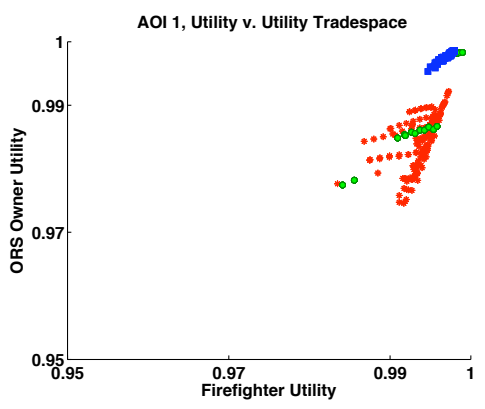

(a) Katrina Disaster

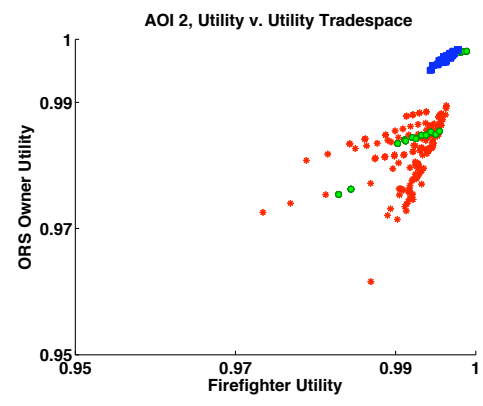

(b) Witch Creek Fire

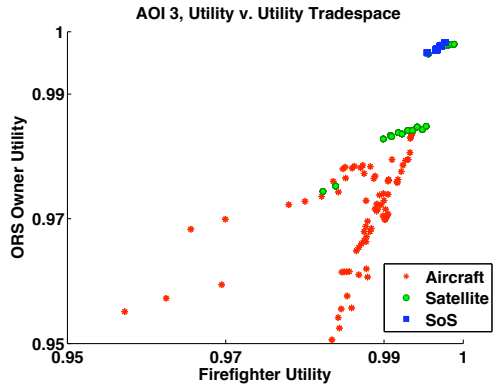

(c) Myanmar Cyclone

Figure 6. Epoch-Era Analysis in SoSTEM with Varying Target Locations, Showing Multiple Single Concepts Along with SoS on the Same Tradespace (AOI = Area of Interest)

\begin{tabular}{|c|c|c|c|c|c|c|c|}
\hline \multirow{2}{*}{$\begin{array}{l}\text { Design } \\
\text { Num }\end{array}$} & \multirow[t]{2}{*}{ Concept } & \multirow[t]{2}{*}{ Description } & \multirow{2}{*}{$\begin{array}{c}\text { Lifetime } \\
\text { Cost }(\$ M)\end{array}$} & \multirow{2}{*}{$\begin{array}{l}\text { Normalized } \\
\text { Pareto } \\
\text { Trace } \\
(\mathrm{N}=3)\end{array}$} & \multicolumn{3}{|c|}{ Pareto Efficient For } \\
\hline & & & & & $\begin{array}{l}\text { Katrina } \\
\text { Disaster }\end{array}$ & $\begin{array}{c}\text { Witch } \\
\text { Creek } \\
\text { Fire }\end{array}$ & $\begin{array}{l}\text { Myan- } \\
\text { mar } \\
\text { Cyclone }\end{array}$ \\
\hline 2116 & Aircraft & existing ScanEagle & 0.7 & 1.00 & Yes & Yes & Yes \\
\hline 2764 & Satellite & $\begin{array}{l}120 \mathrm{~km} \text {, sun-synch } \\
\text { orbit, IR payload }\end{array}$ & 12.4 & 1.00 & Yes & Yes & Yes \\
\hline 925 & SoS & $\begin{array}{l}\text { aircraft (small } \\
\text { UAV w/ piston) + } \\
\text { satellite ( } 800 \mathrm{~km}, \\
\text { sun-synch orbit, } \\
\text { IR payload) }\end{array}$ & 3.862 & 1.00 & Yes & Yes & Yes \\
\hline 1061 & SoS & $\begin{array}{l}\text { aircraft (existing } \\
\text { Cessna } 206)+ \\
\text { satellite }(120 \mathrm{~km} \text {, } \\
23 \text { deg inclination } \\
\text { orbit, IR payload) }\end{array}$ & 3.22 & 0.67 & Yes & Yes & No \\
\hline
\end{tabular}

${ }^{1}$ Normalized Pareto Trace Number is the Pareto Trace Number normalized over the number of epochs considered. In this example, 3 epochs were considered.

Table 2. Selected Pareto Designs for Three Epochs with Change in AOI

The set of Pareto efficient designs that provide high value to both stakeholders can be obtained for each of the three AOI considered. These designs are Pareto efficient for the utility-utility-cost objective, meaning that they are non-dominated designs that are the lowest cost solutions that can maximize the utility perceived by both stakeholders. It may be possible to find designs that are within the Pareto set for all of the AOIs. Other designs may perform well in one AOI, but not in others. Some sample designs from the Pareto sets for the selected AOI are shown in Table 2 to illustrate this point. SoS designs are found in the utility-utility-cost Pareto set as SoS provide high utility, and are comparable cost to some satellites and

$$
9 \text { of } 14
$$


aircraft due to the assumption in this particular scenario that the SoS assets are only partially paid for by the SoS designer (as might happen if the SoS designer pays to lease the component systems owned by other organizations, instead of acquiring them), whereas costs for the aircraft and satellites are assumed to be fully paid by the decision maker (this assumption can be changed to look at other financing options in the future). The first three designs listed in Table 2 are designs that are high performance in all three selected AOI, while the fourth is a valid option if the system were only used for the continental U.S..

From Table 2, it is evident that the SoS tradespace exploration method enables the consideration of a diversity of concepts on the same basis, allowing the designer to consider many different Pareto efficient options that would have been unavailable if conceptual design had been conducted based on a single concept or single mission context.

SoSTEM enables the system designer starting with a large design space to consider many possible options with a relatively small amount of modeling and analysis effort, and then identify a smaller set of value robust designs suitable for further detailed study. The quantitative comparison of a variety of single and multiconcept designs leading to the selection of options for detailed design is in contrast to the simple narrowing of the concept design space early as is sometimes done in traditional systems design. As a result, a larger number of value robust designs can be identified with SoSTEM than with qualitative methods or traditional concept exploration methods alone. While the case used for this study was a hypothetical one, the insights obtained through analysis using this method clearly show that the method can provide valuable information to decision makers who are trying to select value robust designs early in the conceptual design phase.

This case study is discussed in more detail in Chattopadhyay, et al. ${ }^{16}$

\section{B. Satellite Radar System}

The steps of SoSTEM were also applied to the conceptual design of a Satellite Radar SoS, consisting of a Satellite Radar (SR) and airborne radar assets such as JSTARS and Global Hawk. The Satellite Radar System analysis is part of a larger discussion of Intelligence, Surveillance and Reconnaissance (ISR) within the DoD. In a report from the Joint Defense Science Board and the Intelligence Science Board Task Force on Integrating Sensors, ${ }^{17}$ an in-depth discussion of the current state of integration of sensor intelligence, as well as potential future capability gaps is provided. As part of the emphasis on net-centric communications to integrate assets, the DoD is making use of legacy systems in SoS that provide enhanced capabilities for ISR. SoSTEM enables the consideration and comparison of SoS that include the Satellite Radar as a component, assisting decision makers in comparing the merits of a large number of designs. Application of SoSTEM to Satellite Radar may lead to interesting insights that can inform the discussion about this system in the future. Ross, et al. ${ }^{18}$ provides an introduction to the Satellite Radar design problem.

The component systems were classified according to the Managerial Control available to the SoS designer (in this case, the Satellite Radar program manager) over the component systems. The SR program manager is assumed to have complete Managerial Control over the SR component system and a lower level of Managerial Control over the JSTARS and Global Hawk component systems. The SoS attributes and utility information was defined through interviews of proxies for the SR program manager as well as satellite contractor organizations and user proxies. The attributes were categorized into two major sets - attributes focused on imaging-related system performance, and attributes focused on target tracking-related system performance. The target tracking attributes were primary for the initial SoS design, with three of the attributes -target track life, minimum detectable velocity and minimum target radar cross section (RCS) selected as the main attributes for the SoS design. Attributes were mapped to component systems within the SoS in order to define the modeling process.

Each of the component systems was modeled using parametric computer models for new component systems or look-up tables of existing component system performance statistics. The SR component system was modeled in detail using a parametric model relating the SR design variables to the attributes, while the existing JSTARS and Global Hawk system performance was modeled using publicly available performance statistics in those attributes. The SoS consisting of pairs of component systems - SR and JSTARS, and SR with Global Hawk - was modeled first using a low level of attribute combination, and then a medium level of attribute combination each representing a chosen concept of operations for the SoS. In the first case, there is simultaneous operation of the SoS component systems, during which the component system attribute with the best value is chosen as the corresponding SoS attribute performance. This represents a low level of attribute combination complexity, as a selection is made between two simultaneously tracked attributes. A situation like this would potentially arise when the imaging attributes of the SoS are primarily derived from 
the SR and the tracking attributes are derived from the aircraft, such that the best performance between the components is chosen for each SoS attribute. A second option for the concept of operations within the SoS is a hand-off between component systems, in which the SR identifies and begins to track a target, and then hands off the track to the aircraft in the SoS. This concept of operations is represented using a medium level of attribute combination complexity, in which the SoS attribute value is represented by a weighted average of the SR and aircraft attribute values, using the track time as the weighting factor.

The SoS context changes for Epoch-Era Analysis were defined as SoS stakeholder preference changes, changes in availability of component systems, and changes in managerial control and influence over component systems.

Using the component system model of Satellite Radar independently as well as the SoS models consisting of SR and aircraft, tradespace plots comparing traditional single system performance and cost with SoS performance and cost can be generated. Such a tradespace for a particular epoch in which aircraft assets are available for inclusion in the $\mathrm{SoS}$ is shown in Figure 7

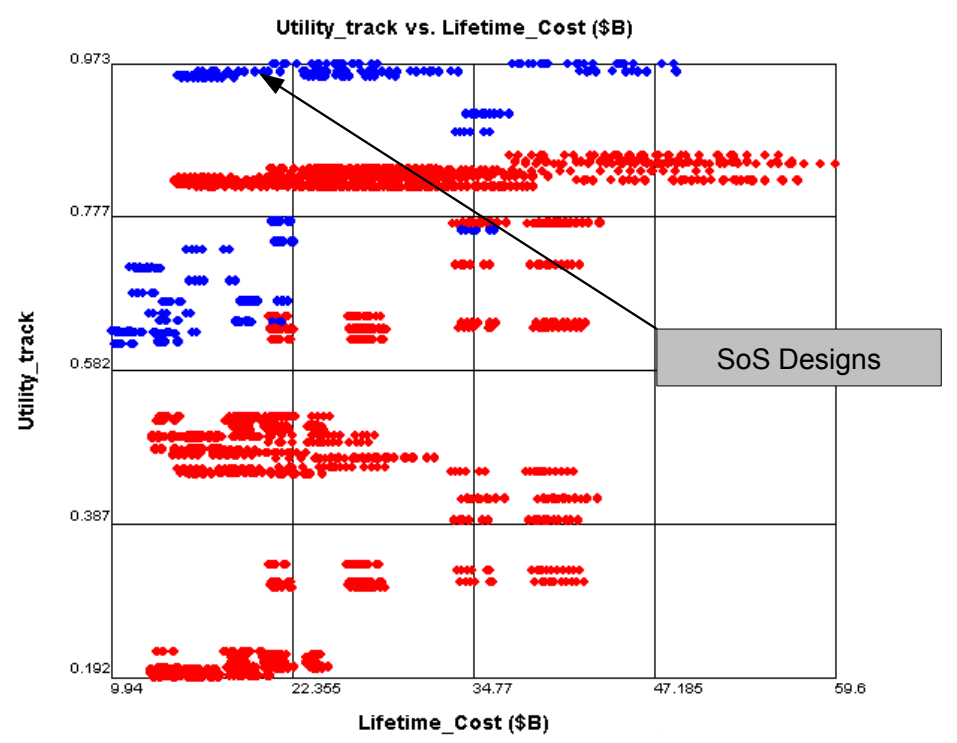

Epoch-Era Analysis can be conducted over several changing epochs with changes in

Figure 7. SoS and Single Space Radar Concepts on Same Tradespace managerial control and stakeholder preference changes. In this case study, 245 epochs were evaluated. Using tradespace statistics such as Normalized Pareto Trace Number (Pareto Trace Number normalized over the number of epochs considered), a set of potentially value robust $\mathrm{SoS}$ designs can be identified. Two example value robust SoS designs from the evaluation of 245 epochs in this case study are given in Table 3.

\begin{tabular}{|c|c|c|c|c|}
\hline Design Num & Concept & Description & $\begin{array}{l}\text { Lifetime } \\
\text { Cost }(\$ B)\end{array}$ & $\begin{array}{l}\text { Normalized } \\
\text { Pareto Trace } \\
(\mathrm{N}=3)\end{array}$ \\
\hline 1718 & SR and Global Hawk & $\begin{array}{l}\text { SR: } 40 \mathrm{~m} \text { antenna, } 1500 \mathrm{~km} \text { alt, } \\
\text { 10/10/1 Walker, } 53 \text { degree inc }\end{array}$ & 14.4 & 1.00 \\
\hline 1944 & SR and JSTARS & $\begin{array}{l}\text { SR: } 100 \mathrm{~m} \text { antenna, } 1500 \mathrm{~km} \\
\text { alt, } 20 / 5 / 1 \text { Walker constella- } \\
\text { tion, } 53 \text { degree inc }\end{array}$ & 45 & 1.00 \\
\hline
\end{tabular}

Table 3. Selected SoS Designs With High Normalized Pareto Trace

Variations in Participation Risk were also studied in this case. Participation Risk is an important consideration for an SoS designer. It may be considered an integral part of the design of the SoS, along with the selection of components, and the interfaces, or the way in which component attributes are combined to form SoS attributes. Effective Managerial Authority of the SoS designer over the various component systems may vary over time, as the availability of incentives changes (e.g., new funds become available for paying off component system managers), or as Managerial Control (MC) changes (e.g., the component system is acquired by the SoS designer, giving the designer maximum control). Examples of changes in 
$\mathrm{MC}$ include going from partial MC in a collaborative SoS, to maximum $\mathrm{MC}$ if the component system is bought by the SoS designer or organization; or going from a low MC to somewhat higher $\mathrm{MC}$ if there is a collaborative partnership developed between the component system organization and the SoS designing organization. Influence can change more readily over time than MC and can be changed by the SoS designer. The ability of a SoS designer to influence a component system may vary with externally defined budgetary variations, if the incentive is in the form of monetary incentives. Influence can also be non-monetary and can come in forms such as useful data made available to the component system, or other benefits that increase the benefit-cost ratio of independent component systems and influence them to participate in the SoS. The combination of MC and Influence provides the Effective Managerial Authority of the SoS designer over the component system, which indicates the likelihood that a component system will participate in the SoS when required. Participation Risk for each of the component systems is estimated from the viewpoint of the SoS designer, in this case, the SR Program Manager. For the purposes of the SoS modeling in this case study, the values for MC and Influence available to the SR Program Manager are allowed to vary over a range of potential values. The SR Program Manager, who is in this case is both a component system decision maker and the SoS designer, has complete managerial control over the SR architecture. Thus MC for the SoS designer over the Satellite Radar component is 1. For this particular case, an example range of MC is used to demonstrate the potential effects of changing MC on design evaluation within the method. To represent a mid-range managerial control for JSTARS, a range of MC values between 0.3 and 0.5 , on a user-defined 0-1 scale (as described in Section V) is chosen. A 0.5 MC value indicates a fifty percent chance that the component system management, in this case, the JSTARS management, will participate in the SoS when needed by the SoS designer. As JSTARS requires a large crew to operate, it is unlikely that the SR program management will be able to acquire such an aircraft for the sole purpose of this surveillance SoS, and will have to collaborate with other organizations for use of the asset. There are also several Global Hawk UAVs owned by the Air Force, that may be used for the SoS. From the managerial viewpoint, it may be easier to gain use of Global Hawks compared to JSTARS for use in the SoS, as Global Hawks are unmanned. In addition, it may be possible for the SR program management to purchase a UAV for full-time use in the SR-aircraft SoS, leading to complete managerial control, or MC of 1 over the Global Hawk component system. Thus the range of SoS Managerial Control selected for Global Hawk in this case study is $0.5-1$. In addition, the SoS designer has some Influence, that he/she can exert over the components. Influence, though determined by the SoS designer, is also considered an epoch constant as it is constrained by budget and other contextual variables in a particular epoch. While Influence is not required for the SR system, due to total managerial control, it is a benefit for determining the behavior of the aircraft assets. In this case also, a range of possible Influence values between 0 and 0.3 is chosen as representative of possible resource availability for the SoS designer. This is a representation of influence, such as monetary or non-monetary incentives, that the SoS designer can provide to the component system management to persuade them to join the SoS. Participation Risk for these AISR assets is then $1-(M C+$ Influence $)$ in each possible case. Given the ranges of Managerial Control (MC) and Influence defined above, several epochs may be defined using the changes in SoS epoch variable managerial control. In addition, the ability to influence the component system behavior is also available to the SoS designer. While Influence is something the SoS designer can control, the ability to change Influence is limited within a given context, and thus Influence available to the SoS designer is treated as a constant within an epoch.

Table 4 shows the generated changes in MC and Influence (In) for each aircraft component system. SR MC is of course 1, and no Influence is needed. The table also shows the resulting PR ${ }^{\natural}$ for each type of SoS.

\begin{tabular}{c|ccc|ccc|ccc|c|c}
\hline Case Num & \multicolumn{3}{|c|}{ SR } & \multicolumn{3}{|c|}{ JSTARS } & \multicolumn{3}{c|}{ Global Hawk } & SR+JSTARS & SR+GH \\
& MC & In & PR & MC & In & PR & MC & In & PR & PR & PR \\
\hline 1 & 1 & 0 & 0 & 0.3 & 0 & 0.7 & 0.5 & 0 & 0.5 & 0.7 & 0.5 \\
2 & 1 & 0 & 0 & 0.3 & 0.3 & 0.4 & 0.5 & 0.3 & 0.2 & 0.4 & 0.2 \\
3 & 1 & 0 & 0 & 0.5 & 0.3 & 0.2 & 1 & 0 & 0 & 0.2 & 0 \\
\hline
\end{tabular}

Table 4. Participation Risk Changes for Different MC and Influence Values

There are several inferences that can be drawn from the PR information presented. For example, in an epoch where the MC over Global Hawk is 1 (e.g., the aircraft is owned by the SoS designer), the PR for a

${ }^{\mathrm{a}}$ Recall $\mathrm{PR}=1-(\mathrm{MC}+$ Influence $)$, and that $P R_{S o S}=1-\left(1-P R_{A}\right)\left(1-P R_{B}\right)$ 
SR+Global Hawk design will be 0, the risk that the SoS will not be available (if it is desired by the SoS designer) is 0. However, in a potential epoch where both JSTARS and Global Hawk are partially controlled, and the SoS designer is allowed limited Influence, either option (given that in earlier analysis, the SoS utility and cost performance was seen to be close) may be good for the SoS designer. In general, designs with low total PR for the SoS are preferred over high PR designs. In addition, designs that have only small variation in PR over many epochs are likely to be stable designs, which will maintain the chosen configuration. However, designs with high variation in PR are potentially problematic over time, with participating SoS components that may leave the SoS with changes in context.

The Satellite Radar case study demonstrates the application of the System of Systems Tradespace Exploration method. The modeling and simulation of SoS using the method enabled the generation of SoS tradespaces on which SoS and single system concepts could be compared on the same performance and cost basis. While this case study represented an example with a small number of components for which SoSTEM could be used, the demonstration of the method on this case study lays the foundation for further applications to designing SoS with larger numbers of components. The preliminary analysis of Participation Risk variations presented here may also be extended further to enable PR to be a system variable indicated on the tradespaces generated using SoSTEM, such that comparisons of SoS designs can be done using criteria of PR, utility and cost simultaneously. As managerial considerations are a key concern in the design of SoS, providing the SoS decision maker with the PR information in conceptual design is likely to enable the selection of more value robust SoS designs.

\section{Discussion}

SoSTEM is a prescriptive, quantitative method for SoS tradespace exploration in the early conceptual design phase. Two applications of this method are discussed in this paper to demonstrate the insights that can be obtained from application of this method to aerospace SoS case studies.

SoSTEM addresses the concept of Managerial Control that the SoS designer has over the component systems, and the resultant Participation Risk of the components in the SoS. As seen in the Satellite Radar case study, the consideration of Participation Risk along with the traditional utility and cost metrics in the evaluation and comparison of SoS designs provides the decision maker with insight into the managerial concerns that may arise during implementation and operation of a chosen design. Thus SoSTEM not only enables the decision maker to consider system performance but also the effect of the independent component system management decisions on the overall SoS performance over the system lifetime. SoSTEM also includes a framework to combine component system attributes to obtain the SoS attributes in order to quantitatively model SoS performance. SoSTEM enables the tradespace exploration of diverse SoS on the same tradespace along with single systems, allowing the decision maker to more completely explore the available design tradespace. Using this method, value robust SoS designs can be identified as candidates for detailed design.

SoSTEM is intended as a method for early conceptual design. It is devised as a framework for exploring the variety of SoS related issues in early conceptual design that will allow the SoS decision makers to make broad design trades in situations where not all of the information about the SoS is available. The method can be used iteratively as further information about variables like the Participation Risk of a component system, or more detailed performance models become available, or as context variables change. Thus there is potential for this method to also be used in the operations phase of a SoS, to study the change in SoS value delivery when changes in context or composition of the SoS occur. A more detailed discussion of many of the concepts presented in this paper, along with areas for future development of the method, is available in the Master's thesis by Chattopadhyay. ${ }^{19}$

\section{Conclusion}

Systems of Systems are of increasing importance as organizations seek to leverage multiple existing systems in order to develop new capabilities. Leveraging existing systems, and using new systems to bridge capability gaps, is an appealing approach in an era of limited funding for large engineering projects. Thus emphasis on effective SoS design methods to aid decision makers is presently a key approach in systems engineering research. The System of Systems Tradespace Exploration Method (SoSTEM) discussed in this paper addresses some of the issues faced by SoS design engineers in creating planned SoS that achieve defined goals, and enables SoS designers to systematically work through many of the challenges of SoS 
design. This quantitative tradespace exploration method can be used in conceptual design of SoS to enable the comparison of a large number of design alternatives on the same tradespace, as well as to study the changes in the value delivery of SoS over changing contexts as is demonstrated in two case studies presented in this paper. SoSTEM can thus be used as a conceptual design tool by decision makers in selecting value robust SoS designs.

\section{Acknowledgements}

Funding for this research was provided by the Systems Engineering Advancement Research Initiative (SEAri), a research initiative within the Engineering Systems Division at the Massachusetts Institute of Technology. SEAri (http://seari.mit.edu) brings together a set of sponsored research projects and a consortium of systems engineering leaders from industry, government, and academia. SEAri gratefully acknowledges the support of the Charles Stark Draper Laboratory and the U.S. Government in this research.

\section{References}

${ }^{1}$ INCOSE, "Systems Engineering Handbook v.3: A Guide for System Life Cycle Processes and Activities," Tech. rep., June 2006.

${ }^{2}$ Maier, M. W., "Architecting Principles for Systems-of-Systems," Systems Engineering, Vol. 1, No. 4, 1998, pp. $267-284$.

${ }^{3}$ Sage, A. P. and Cuppan, C. D., "On the Systems Engineering and Management of Systems of Systems and Federations of Systems," Information Knowledge Systems Management, Vol. 2, No. 4, 2001, pp. 325-345.

${ }^{4}$ Boardman, J. and Sauser, B., "System of Systems - the meaning of OF," IEEE International Systems Conference, Los Angeles, CA, 2006.

${ }^{5}$ Eisner, H., Marciniak, J., and McMillan, R., "Computer-Aided System of Systems (S2) Engineering," Vol. 1, IEEE International Conference on Systems, Man and Cybernetics, Charlottesville, VA, October 1991, pp. 531-537.

${ }^{6}$ Keating, C., Rogers, R., Unal, R., Dryer, D., Sousa-Poza, A., Safford, R., Peterson, W., and Rabadi, G., "System of Systems Engineering," Engineering Management Journal, Vol. 15, No. 3, 2003, pp. 36-45.

${ }^{7}$ Carlock, P. G. and Fenton, R. E., "System of Systems (SoS) Enterprise Systems Engineering for Information-Intensive Organizations," Systems Engineering, Vol. 4, No. 4, June 2001, pp. 242-261.

${ }^{8}$ DeLaurentis, D. A., "Understanding Transportation as a System-of-Systems Design Problem," AIAA Aerospace Science Meeting Exhibit, 2005.

${ }^{9}$ Ross, A. M., Managing Unarticulated Value: Changeability In Multi-Attribute Tradespace Exploration, Ph.D. thesis, Massachusetts Institute of Technology, 2006.

${ }^{10}$ Ross, A. M. and Rhodes, D. H., "The System Shell as a Construct for Mitigating the Impact of Changing Contexts by Creating Opportunities for Value Robustness," IEEE Systems Conference, Honolulu, HI, 2007.

${ }^{11}$ Chattopadhyay, D., Ross, A. M., and Rhodes, D. H., "A Framework for Tradespace Exploration of Systems of Systems," Conference on Systems Engineering Research, Los Angeles, CA, April 2008.

${ }^{12}$ Ross, A. M. and Rhodes, D. H., "Using Attribute Classes to Uncover Latent Value During Conceptual Systems Design," IEEE International Systems Conference, Montreal, Canada, April 2008.

${ }^{13}$ Richards, M. G., Ross, A. M., Shah, N. B., and Hastings, D. E., "Metrics for Evaluating Survivability in Dynamic Multi-Attribute Tradespace Exploration," AIAA Space, San Diego, CA, September 2008.

${ }^{14}$ Hall, D. L. and Llinas, J., "An Introduction to Multisensor Data Fusion," Proceedings of IEEE, Vol. 85, IEEE, January 1997, pp. 6-23.

${ }^{15}$ Ross, A. M., Rhodes, D. H., and Hastings, D. E., "Using Pareto Trace to Determine System Passive Value Robustness," IEEE International Systems Conference, Vancouver, Canada, March 2009.

${ }^{16}$ Chattopadhyay, D., Ross, A. M., and Rhodes, D. H., "Demonstration of System of Systems Multi-Attribute Tradespace Exploration on a Multi-Concept Surveillance Architecture," Conference on Systems Engineering Research, Loughborough, UK, April 2009.

${ }^{17}$ Defense Science Board, "Integrating Sensor-Collected Intelligence," Tech. rep., Joint Defense Science Board Intelligence Science Board Task Force Office of the Under Secretary of Defense for Acquisition, Technology and Logistics, Pentagon, Washington, DC, December 2008.

${ }^{18}$ Ross, A. M., McManus, H. L., Long, A., Richards, M. G., Rhodes, D. H., and Hastings, D. E., "Responsive Systems Comparison Method: Case Study in Assessing Future Designs in the Presence of Change," AIAA Space 2008 Conference and Exposition, San Diego, CA, September 2008.

${ }^{19}$ Chattopadhyay, D., A Method for Tradespace Exploration of Systems of Systems, Master's thesis, Massachusetts Institute of Technology, June 2009. 